\title{
Extraction of Human Stepping Pattern Using Acceleration Sensors
}

\author{
Takayuki Toyohira ${ }^{*}$, Kiminori Sato ${ }^{1}$ and Mutsumi Watanabe $^{1}$ \\ ${ }^{1}$ Graduate School of Science and Engineering, Kagoshima University, Kagoshima,890-0065, Japan
}

\begin{abstract}
Gait analysis plays an important role in characterizing individuals and each condition and gait analysis systems have been developed using various devices or instruments. However, most systems do not catch synchronous stepping actions between right foot and left foot. For obtaining a precise gait pattern, a synchronous walking sensing system is developed, in which a pair of acceleration and angular velocity sensors are attached to left and right shoes of a walking person and their data are transmitted to a PC through a wireless channel. Walking data from 19 persons of the age of 14 to 20 are acquired for walking analysis. Stepping time diagrams are extracted from the acquired data of right and left foot actions of stepping-off and-on the ground, and the time diagrams distinguish between an ordinary person and a person injured on left leg, and a stepping recovery process of the injured person is shown. Synchronous sensing of stepping action between right foot and left foot contributes to obtain precise stepping patterns.
\end{abstract}

\section{Introduction}

Gait pattern of person characterizes individual and each condition. The precise gait pattern may be one of the measures for recognizing individuals [1][2][3][4]. Gait analysis systems using acceleration sensor were developed for medical rehabilitation [5] and identification of portable device user [6], etc. However, most systems do not catch synchronous stepping actions between right foot and left foot. In this paper, a synchronous walking sensing system is developed, where a pair of acceleration and angular velocity sensors are attached to left and right shoes of a walking person and their data are transmitted to a PC through a wireless channel. Walking data from 19 students of the age of 14 to 20 are acquired for walking analysis. From acquired data, stepping time diagrams are extracted and the difference of stepping patterns between an ordinary person and a person injured on left leg and the recovery process of the injured person are shown.

\section{Human walking}

Human is an only creature who moves by erect bipedal walking. Always birds as ostrich and penguin and temporarily animals as dog, bear, monkey and ape move by bipedal walking, but they cannot move in an erect posture. Human walking action is classified into walk and

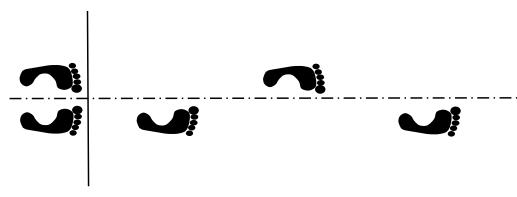

Fig. 1. Feet touch positions of walking movement. trot [7]. Usually human walking features trot in which pivot foot alternately changes and either foot touches on the ground. Fig. 1 shows an example of surface touchdown positions of left and right feet when a person stands and starts walking from the right step and moves forward.

A trot action is regarded as a rotation movement around the touched ground or floor. In this case, the rotation energy does not exceed the gravity energy, as follows:

$$
m v^{2} / r \leq m g
$$

where $v, r$ and $m$ are the walking velocity, the gravity centre and the mass of a person, and the acceleration of the gravity is $g=9.80\left[\mathrm{~m} / \mathrm{s}^{2}\right]$. Then the maximum value of trotting velocity is obtained as

$$
v_{\max }=\sqrt{r g} \text {. }
$$

For the height $r=1[\mathrm{~m}]$ of the gravity centre of a person, the trotting velocity is limited to $v_{\max }=3.13[\mathrm{~m} / \mathrm{s}]$.

If the moving velocity of a person exceeds $v_{\max }$, the moving action becomes running. In the case of trot, the horizontal momentum is delivered to the next action at a full efficiency, and the vertical momentum disappears by collision between the foot and the ground, while the gravity energy of the gravity centre is preserved by Achilles' tendon and delivered to the next action by kicking the ground. In the case of walk, the weight is supported by both feet and the velocity vector of the gravity centre is restricted toward one way and the left and right momentum by zigzag movement is vanished in

* Corresponding author: toyohira@kagoshima-ct.ac.jp 
addition to the vertical momentum and so the energy efficiency of walk is inferior to trot.

In this paper, we use the term of walking for the moving action which mainly consists of trot rather than walk. Real walking accompanies with so complicated actions interlinked by feet, leg, body and moving velocity, etc, that walking patterns can be utilized to identification of individuals and each condition.

\section{Walking data acquisition system}

Fig.2 shows a walking data acquisition system using a couple of acceleration and angular velocity sensors. A person taking a pair of shoes attached with sensors moves by walking at a flat ground or floor, while the respective sensor transmits data to a PC through Bluetooth wireless channel. Table 1 shows specifications of the sensor TSND121 which detects 3-axes accelerations and 3-axes angular velocities and additionally earth magnetism and atmospheric pressure. The PC controls sensors by instructions of start and stop and processes the acquired data of 6-axes accelerations and angular velocities. Fig.3 shows an overview of the sensor in comparison with a $500 y e a n$ coin of $2.65 \mathrm{~cm}$ diameter, where $\mathrm{x}, \mathrm{y}$ and $\mathrm{z}$ axes in acceleration sensing are drawn on the surface. Fig.4 shows directions of rotation angles around $\mathrm{x}, \mathrm{y}$ and $\mathrm{z}$ axes in angular velocity sensing. Fig. 5 shows a picture of shoes attached to the sensors with $\mathrm{x}$ axis of acceleration directed forward. The sensors are attached firmly to the shoes at the stable position [8][9].

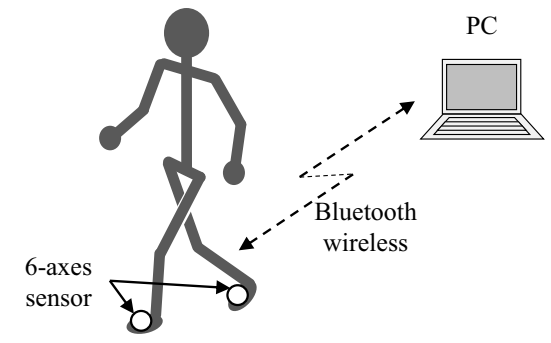

Fig.2. Walking data acquisition system.

Table 1. Specifications of sensor.

\begin{tabular}{l|l}
\hline $\begin{array}{l}\text { Acceleration sensor } \\
\text { Sampling rate }\end{array}$ & InvenSense MPU-6050 \\
Accelerometer Feature & $\pm 2 \mathrm{G}, \pm 4 \mathrm{G}, \pm 8 \mathrm{G}$ and $\pm 16 \mathrm{G}$ \\
Gyroscope Feature & $\pm 250 \mathrm{dps}, \pm 500 \mathrm{dps}, \pm 1000 \mathrm{dps}$ \\
& and $\pm 2000 \mathrm{dps}$ \\
\hline Magnetic Field sensor & Aichi Steel AMI306 \\
\hline Barometer & Freescale MPL3115A2 \\
\hline
\end{tabular}

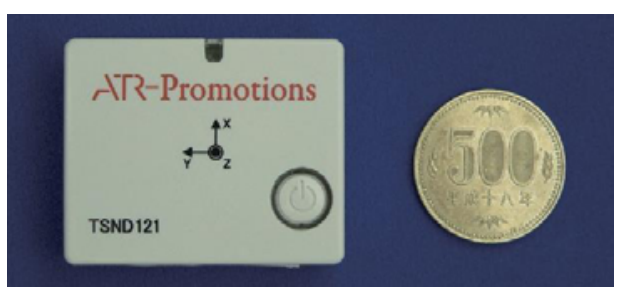

Fig. 3. Overview of sensor.



Fig.4. Angular directions in angular velocity sensing.

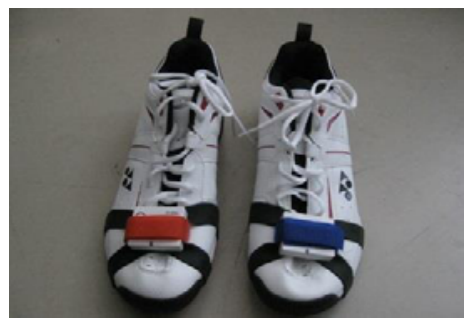

Fig.5. Shoes attached to sensors.
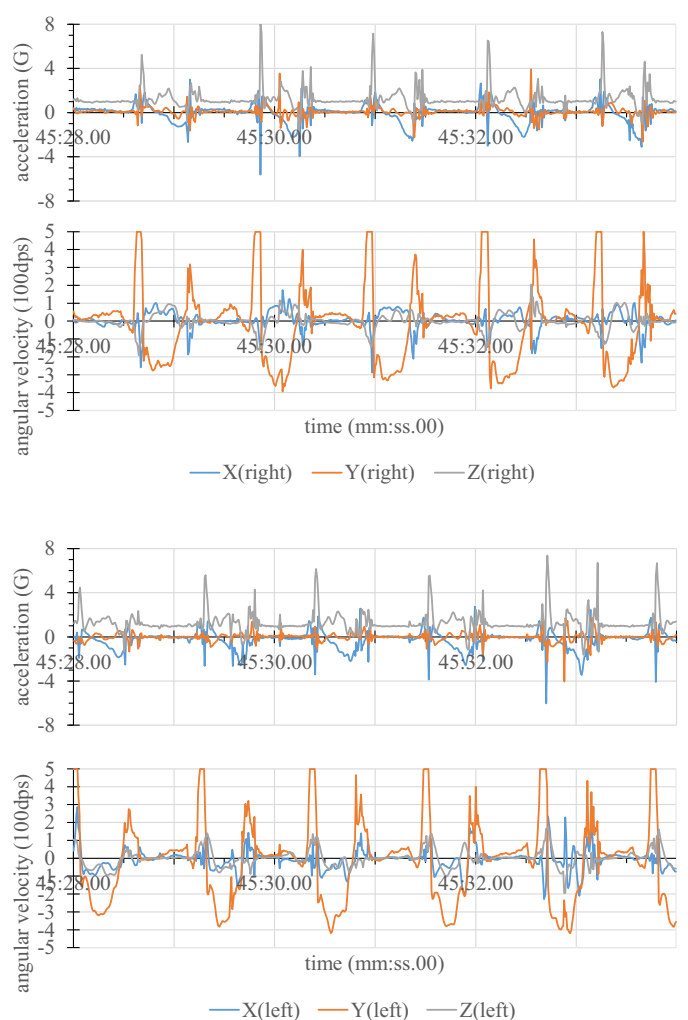

Fig.6. Acceleration and angular velocity responses for an ordinary person.

\section{Data acquisition of walking}

At the floor of the college building, walking data were acquired from 19 male and female students of the age of 14 to 20 , one of whom had been injured on left leg but could join in the experiments. The walking manner is explained as follows: a subject person stands at the start point and raises both tiptoes in order to synchronize between left and right sensors and then begins to walk 


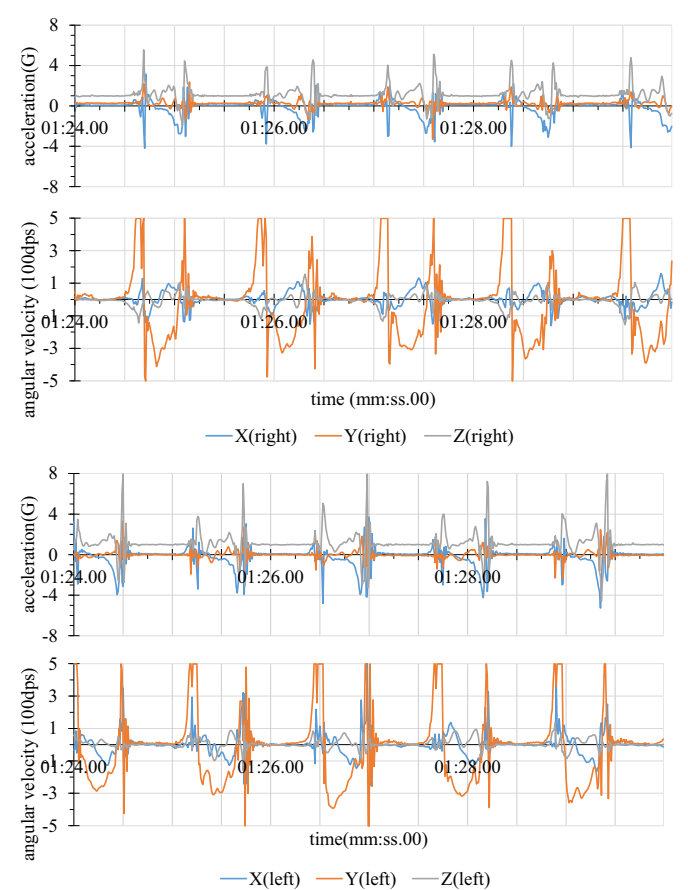

Fig.7. Acceleration and angular velocity responses for an injured person

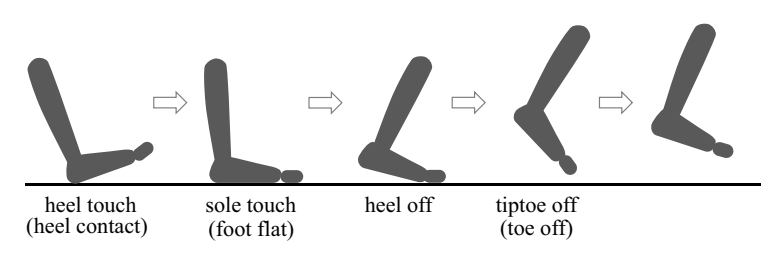

Fig.8. Stepping action of a foot.

from the right or left foot. Fig. 6 shows partial waveforms of $\mathrm{x}, \mathrm{y}$ and $\mathrm{z}$ components of acceleration and angular velocity on right foot and left foot of an ordinary person, and Fig. 7 shows those of a person injured on left leg, where each waveform indicates almost steady response after initial irregular response. The responses for the injured person seem to be more irregular than those for the ordinary person. In these responses, the stepping order is indicated by numbering and the marked time positions of $\mathrm{x}$ and $\mathrm{z}$ components of accelerations and $\mathrm{y}$ component of angular velocity are regarded as unique changing points of stepping off and on the ground [10]. We consider that the negatives short pulse of $x$ acceleration and the positive short pulse of $\mathrm{z}$ acceleration occurs simultaneously with the positive to negative rebound pulse of $y$ angular velocity and so the time when the stepping-on changes to stepping-off is the time on the peaks of $\mathrm{x}$ and $\mathrm{z}$ acceleration pulses and the zero-crossing of $y$ angular velocity rebound pulse. And we consider that the negative pulses of $\mathrm{x}$ and $\mathrm{z}$ accelerations occurs simultaneously and so the time when the stepping-off changes to stepping-on is the time on the peaks of $x$ and $z$ acceleration negative pulses. The above stepping-off and -on interpretations are supported by the foot actions of Fig. 8 . There, first a heel touches on the ground, a sole adheres to the ground, the heel rises with a tiptoe on the ground, and at last the tiptoe rises off the ground with the heel. The first action gives

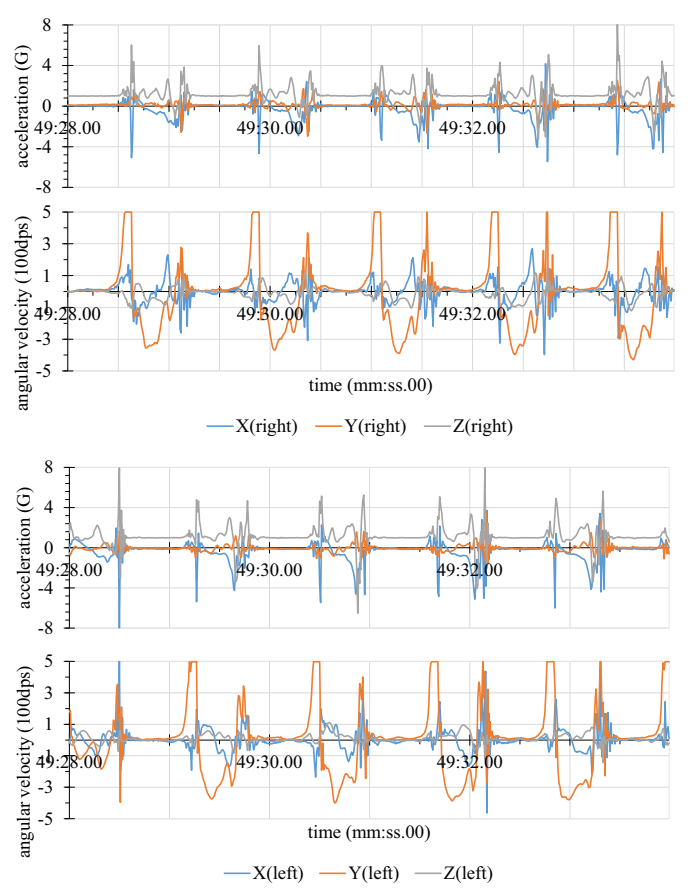

Fig.9. Acceleration angular velocity responses for the injured person at the second experiment.
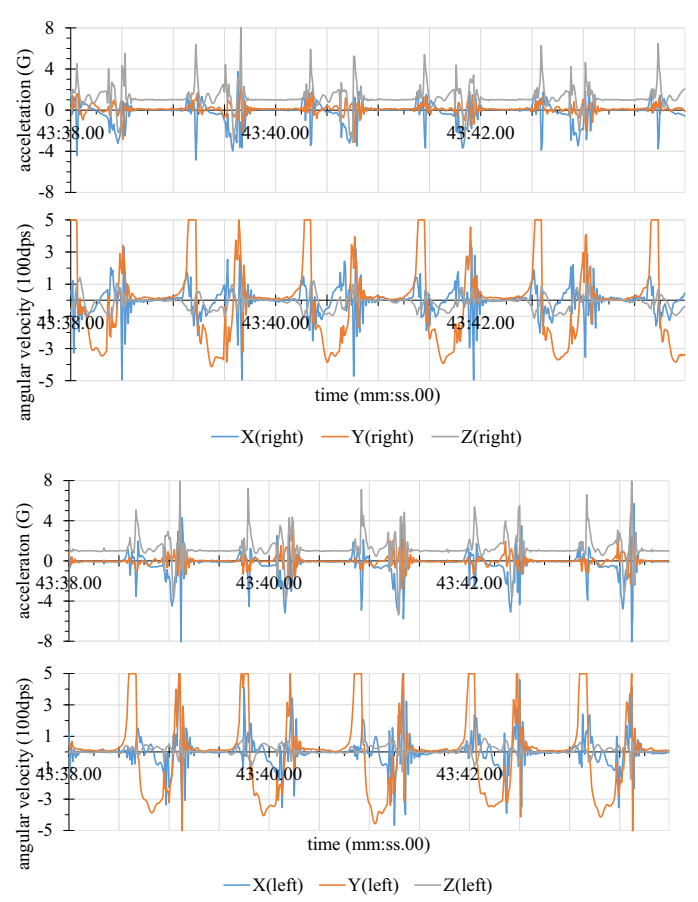

Fig.10. Acceleration and angular velocity responses for the injured person at the third experiment.

downward $\mathrm{x}$ and $\mathrm{z}$ accelerations and the last action gives the rebound rotation around y axis. Fig. 9 and Fig.10 show $\mathrm{x}$ and $\mathrm{z}$ components of acceleration and $\mathrm{y}$ components of angular velocity for the injured person at the second experiment and the third experiment, respectively, at the interval of two weeks. 


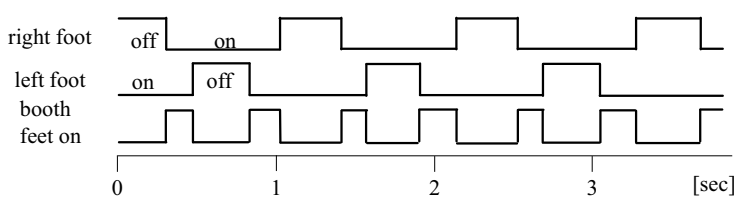

Fig.11 Stepping time diagram for the ordinary person.

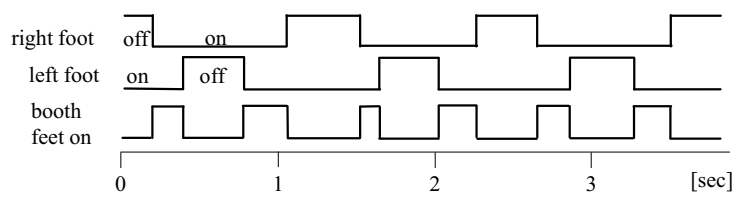

Fig.12. Stepping time diagram for the injured person.

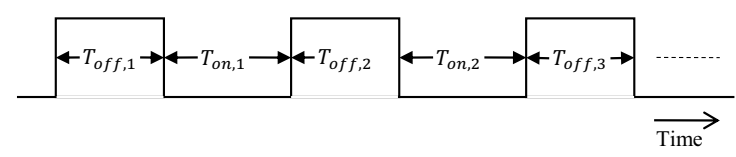

Fig.13. Stepping-off and-on time intervals.

Table 2. Statistics of time intervals of stepping-off and-on.

\begin{tabular}{|c|c|c|c|c|c|c|}
\hline & \multicolumn{3}{|c|}{ Ordinary person } & \multicolumn{3}{|c|}{ Injured person } \\
\hline & Right foot & Left foot & Both feet & Right foot & Left foot & Both feet \\
\hline$\overline{\bar{T}_{\text {off }}}$ & 0.395 & 0.367 & & 0.379 & 0.378 & \\
\hline$\sigma_{0, \pi}$ & 0.015 & 0.030 & & 0.031 & 0.017 & \\
\hline $\bar{T}_{\text {or }}$ & 0.759 & 0.790 & & 0.828 & 0.830 & \\
\hline$\sigma_{o n}$ & 0.038 & 0.046 & & 0.034 & 0.018 & \\
\hline$T_{\text {on-nger-lept }}$ & & & 0.182 & & & 0.212 \\
\hline$\sigma_{\text {on-mgan-ient }} \mid$ & & & 0.021 & & & 0.028 \\
\hline$T_{\text {on-lept-myts }}$ & & & 0.214 & & & 0.238 \\
\hline$\sigma_{\text {on } I f t}$ right|r| & & & 0.036 & & & 0.013 \\
\hline
\end{tabular}

\section{Evaluation of stepping time diagrams}

In order to evaluate the stepping actions, we extract the time intervals of stepping off and on the ground from the acceleration and angular velocity responses. As mentioned in the above chapter, from the unique changing points in Fig.6 and Fig.7, we obtain the stepping time diagrams of the right and left feet. Fig. 11 and Fig. 12 show the stepping time diagrams of the ordinary person and the injured person, respectively, where another time diagram illustrates the time intervals simultaneously stepping on the ground. The simultaneously stepping-on time diagram of the ordinary person is more balanced than that of the injured person. The simultaneous stepping-on occurs two times in a pair of the right and left steps. The stepping-off and-on time intervals are evaluated by a model of Fig.13, where $T_{o f f, k}$ is stepping-off time interval and $T_{o n, k}$ is stepping-on time interval. The respective $\mathrm{N}$ intervals give the average values

$$
\begin{aligned}
& \bar{T}_{\text {off }}=(1 / N) \sum_{k=1}^{N} T_{o f f, k} \\
& \bar{T}_{o n}=(1 / N) \sum_{k=1}^{N} T_{o n, k}
\end{aligned}
$$

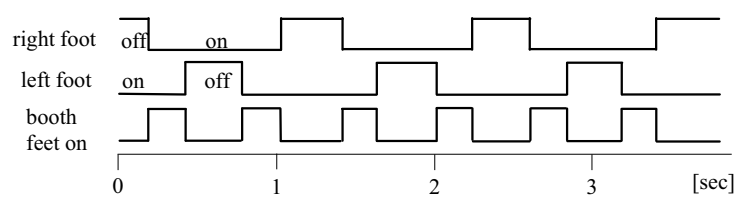

Fig.14. Stepping time diagram of 2nd experiment for the injured person.

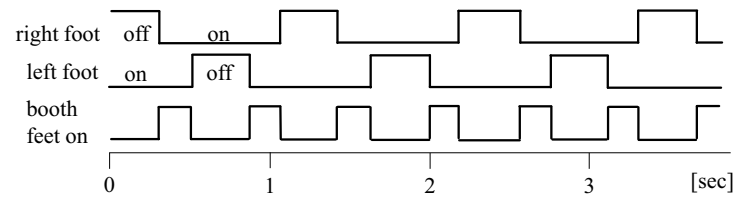

Fig.15.Stepping time diagram of 3rd experiment for the injured person.

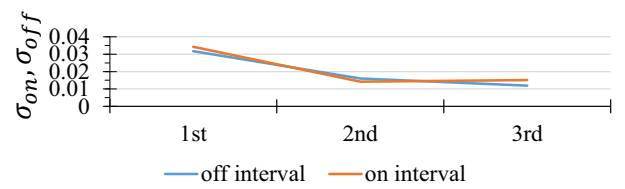

Fig.16. Standard deviations of right foot stepping time interval in second.

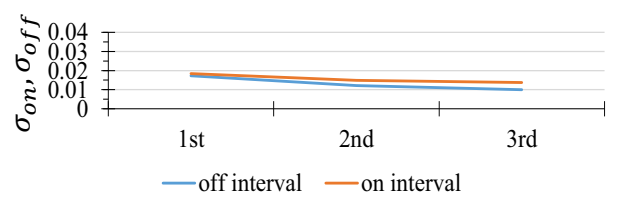

Fig.17. Standard deviations of left foot stepping time interval in second.

and the variances

$$
\begin{aligned}
& \sigma_{o f f}^{2}=(1 / N) \sum_{k=1}^{N}\left(T_{o f f, k}-\bar{T}_{o f f}\right)^{2} \\
& \sigma_{o n}^{2}=(1 / N) \sum_{k=1}^{N}\left(T_{o n, k}-\bar{T}_{o n}\right) .^{2}
\end{aligned}
$$

From the time intervals in Fig.11 and Fig.12, we obtain the average values and the standard deviations of stepping-off and -on time intervals of the right foot and the left foot for the ordinary person and for the injured person. Similarly, we define the simultaneously steppingon time intervals and obtain the average values and standard deviation of the simultaneously stepping-on time intervals for the ordinary person and for the injured person Table 2 shows the average values and the standard deviations of these time intervals in second. Fig.14 and Fig.15 shows the stepping-off and -on time diagrams derived from Fig.9 and Fig.10. From the time intervals in Fig.12, Fig. 14 and Fig.15 for the injured person, we obtain the average value and the standard deviations of the stepping-off and stepping-on time intervals of the 1st, 2nd and 3rd experiments of the right foot as $\bar{T}_{\text {off }, n, \text { right }}$, $\bar{T}_{\text {on,n,right }}, \sigma_{o f f, n, r i g h t}, \sigma_{\text {on,n,right }}$ and those of the left foot as $\bar{T}_{\text {off }, n, \text { left }}, \bar{T}_{\text {on,n,left }}, \sigma_{\text {off,left }, n}, \sigma_{\text {on,n,left }}$ 


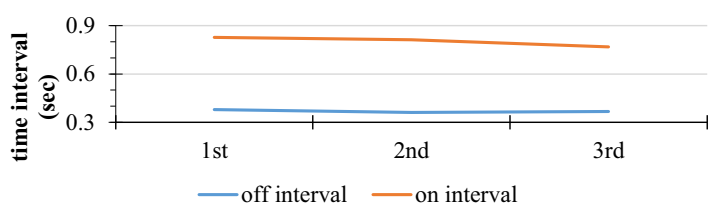

Fig.18. Average values of right foot stepping time interval.

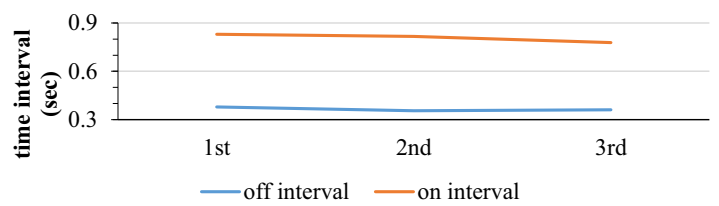

Fig.19. Average values of left foot stepping time interval.

( $n=1,2,3, N=11)$. Fig.16 and Fig.17 show convergence process of standard deviations of stepping-off and stepping-on time intervals of the right foot and the left foot, respectively. Fig.16 and Fig.17 show that the stepping action of the injured person recovers and become stable according to time progress. Fig. 18 and Fig. 19 show convergence process of average values of stepping-off and stepping-on time intervals. From the 3 rd values of $T_{o f f, 3, r i g h t}=0.367[\mathrm{~s}] \quad, \quad T_{o n, 3, \text { right }}=0.769[\mathrm{~s}] \quad$ and $T_{\text {off } 3, \text { left }}=0.360[\mathrm{~s}], T_{\text {on,3,left }}=0.779[\mathrm{~s}]$, we obtain the average step time interval $\left(T_{\text {off }, 3, \text { right }}+T_{\text {off }, 3, \text { left }}+T_{\text {on, }, 3, \text { right }}+T_{\text {on, } 3, \text { right }}\right) / 2=1.14[\mathrm{~s}]$.

Moreover, we obtain the average values and the standard deviations of the simultaneously stepping-on time intervals at the 1st, 2nd and 3rd stages for the injured person, as $T_{\text {on-right-left }, 1}=0.212[\mathrm{~s}], T_{\text {on-left-right, } 1}=0.238[\mathrm{~s}]$, $T_{\text {on-rightleft }, 2}=0.234[\mathrm{~s}] \quad, \quad T_{\text {on-left }- \text { right }, 2}=0.234[\mathrm{~s}] \quad$, $T_{\text {on-right-left }, 3}=0.222[\mathrm{~s}], T_{\text {on-left-right }, 3}=0.190[\mathrm{~s}] . \quad$ From these results, the stepping pattern of the injured person approaches those of the ordinary person.

\section{Conclusions}

A walking data acquisition system using a couple of acceleration and angular velocity sensors attached to each foot is developed. The system is applied to acquisition of walking data from 19 male and female students including a male student in the recovery process of injured left leg. In the sensor data, $\mathrm{x}$ and $\mathrm{z}$ components of accelerations and y component of acceleration are used for analysis of walking. The stepping-off and -on time diagrams characterizes the walking of the ordinary student and injured student, and gives a recognition of the recovery process of the injured student. Synchronous sensing of stepping action between right foot and left foot contributes to obtain precise stepping patterns. The other data of accelerations and angular velocities may be used for the recognitions of individual and each condition in the future.

\section{Acknowledgement}

The authors appreciate greatly the helpful suggestions of Dr. Yoshihiro Tanada of Kyoto University and students' cooperation and assistance of the experiments.

\section{References}

1. J. Han, B. Bhanu, Individual Recognition Using Gait Energy Image, Trans. on Pattern Analysis and Machine Intelligence, Vol. 28, No.2, pp. 316-322 (2006).

2. Y. Makihara, R. Sagawa, Y. Mukaigawa, T. Echigo, Y. Yagi, Gait Recognition Using a View Transformation Model in the Frequency Domain, Proc. of the 9th European Conf. on Computer Vision, Graz, Austria, pp. 151-163 (2006).

3. K. Bashir, T. Xiang, S. Gong, Gait recognition without subject cooperation, Pattern Recognition Letters, Vol.31, No. 13, pp.2052-2060 (2010).

4. T. H. W. Lam, K. H. Cheung, J. N. K. Liu, Gait flow image: A silhouette-based gait representation for human identification, Pattern Recognition, Vol.44, pp. 973-987 (2011).

5. T. Kobatyashi, Y. Miyake, Y. Wada, M. Matsubara, Kinematic Analysis System of Walking by Acceleration Sensor, Trans. Inst. Auto. Contl. Japan, Vol.42, No.5(2006).

6. J. Manyjarvi, M. Lindholm, E.Vildjounaite, S. Makela, H.Alisto, Identifying Users of Portable Devices from Gait Pattern with Accelerometers, ICAPSSP'05, March (2005).

7. R. M. Alexander, Principles of Animal locomotion, Princeton University Press (2003).

8. Y. Ohtaki, K. Sagawa, H. Inooka, A Method for the Long-Term Gait Assessment Utilizing Accelerometers and Gyroscopes, Trans. of the JSME (in Japanese), Series C, Vol. 67, No. 655, pp. 192-198 (2001).

9. A. R. Jimenz, F. Seco, C Prieto, J. Guevara, A Comparison of Pedestrian Dead-Reckoning Algorithms using a Low-Cost MEMS IMU, In Proceedings of Intelligent Signal Processing, pp.37-42 (2009)

10. K. Sagawa, Y. Sato, H. Inooka, Non-Restricted Measurement of Distance during Level Walk, Trans. of the SICE, Vol. 36, No. 11, pp. 909-915 (2000). 